\title{
Organic Amine-Mediated Synthesis of Polymer and Carbon Microspheres: Mechanism Insight and Energy-related Applications
}

Jitong Wang, Liwen Yao, Cheng Ma, Xuhong Guo, Wenming Qiao, Licheng Ling and Donghui Long*

State Key Laboratory of Chemical Engineering, East China University of Science and Technology,

Shanghai 200237, China

* Corresponding author: Donghui Long, Tel: +86-21-64252924. Fax: +86-21-64252914.

E-mail: longdh@mail.ecust.edu.cn 


\section{Content:}

Table S1. Chemical composition measured by elemental analysis and theoretical composition of PMSs.

Table S2. Zeta potential of PEI and R-PEI solution with different molecular weights.

Table S3. The relative contents of different functionalities in N 1s peaks from XPS spectra.

Figure S1. Particle size distributions of polymer microspheres synthesized with PEI-1800.

Figure S2. SEM images of PMS-PEI-600 obtained at different molar ratio of PEI/R: (a) 0.5; (b) 1; (c) 1.5; different concentration: (d)3 wt. \%; (e)5 wt. \%; (f)7 wt. \%; different temperature: (g)30 ${ }^{\circ} \mathrm{C}$; (h) $50{ }^{\circ} \mathrm{C}$; (i) $70{ }^{\circ} \mathrm{C}$.

Figure S3. FT-IR spectra (a) and XPS survey (b) and high-resolution N 1s (c-f) spectra for PMSs synthesized of PEI with different molecular weight; (c) PMS-1; (d) PMS-2; (e) PMS-3 and (f) PMS-3.

Figure S4. A digital photo image of the solution obtained with different molecular weight of PEI. a. PMS-1; b. PMS-2; c. PMS-3 and d. PMS-4; (a) after 30 min; (b) after $72 \mathrm{~h}$.

Figure S5. Transient changes of the hydrodynamic size of colloidal particles synthesized with TEPA.

Figure S6. Transient changes of the hydrodynamic size of colloidal particles synthesized with TEOS using conventional Stöber synthesis method.

Figure S7. TEM images of PMS-4 (a) and CMS-4 (b).

Figure S8. XPS survey (a) and high-resolution N 1s (b-d) spectra for CMSs synthesized from branched PEI;

(b) CMS-1, (c) CMS-3 and (c) CMS-4.

Figure S9. XPS survey (a, b) and high-resolution N 1s (c, d) spectra for ACMSs synthesized with branched PEI; (a, c) ACMS3-2 and (b, d) ACMS3-3.

Figure S10. Galvanostatic charge/discharge voltage profiles at different current densities for ACSM3-2 and ACSM3-3. 
Figure S11. Nyquist plots and the enlargement of the high-frequency region (insert) for CMS-3, ACMS3-2 and ACMS3-3.

Figure S12. (a) cyclic voltammetry (CV) testing at $100 \mathrm{mV} \cdot \mathrm{s}^{-1}$; (b) Galvanostatic charge/discharge voltage profiles at $0.1 \mathrm{~A} \cdot \mathrm{g}^{-1} ;$ (c) Plot of specific capacitances calculated from the discharge curves versus current density; (d) Cycling ability at $0.1 \mathrm{~A} \cdot \mathrm{g}^{-1}$ for CMS-3, ACMS3-2 and ACMS3-3. 
Table S1. Chemical composition measured by elemental analysis and theoretical composition of PMSs

\begin{tabular}{|c|c|c|c|c|c|}
\hline \multirow[b]{2}{*}{ Samples } & \multicolumn{4}{|c|}{ Elements analysis } & \multirow{2}{*}{$\begin{array}{l}\text { theoretical } \\
\text { nitrogen } \\
\text { content } \\
\text { (wt. \%) }\end{array}$} \\
\hline & $\begin{array}{c}\mathrm{N} \\
(\text { wt. \%) }\end{array}$ & $\begin{array}{c}\text { C } \\
\text { (wt. \%) }\end{array}$ & $\begin{array}{c}\mathrm{O} \\
(\text { wt. \%) }\end{array}$ & $\begin{array}{c}\mathrm{N} / \mathrm{C} \\
\text { (at./at.) }\end{array}$ & \\
\hline EDA & 7.3 & 56.6 & 30.2 & 0.11 & 9.0 \\
\hline DETA & 8.1 & 53.0 & 32.7 & 0.13 & 8.9 \\
\hline TEPA & 9.2 & 56.1 & 27.9 & 0.14 & 8.8 \\
\hline HEDA & 7.1 & 56.8 & 29.4 & 0.11 & 7.6 \\
\hline $\mathrm{PA}$ & 8.7 & 59.6 & 25.0 & 0.13 & 8.4 \\
\hline PS3 & 9.8 & 57.7 & 25.1 & 0.15 & 8.6 \\
\hline
\end{tabular}

The CHN elemental results of polymer microspheres synthesized from various amines are measured and the theoretical nitrogen values are calculated based on the precursor chemistry. The results shows that the obtained nitrogen contents in the microspheres are very close to the theoretical values, which means all these organic amines could be well involved in the polymerization reaction and induce the formation of microspheres. 
Table S2. Zeta potential of PEI and R-PEI solution with different molecular weights

\begin{tabular}{ccccc}
\hline Zeta potential & \multicolumn{3}{c}{ molecular weight (Mw) } \\
\cline { 2 - 4 }$(\mathrm{mV})$ & 600 & 1200 & 1800 & 10000 \\
\hline PEI & -2.36 & -3.41 & -0.57 & -1.65 \\
R-PEI & 7.03 & 4.58 & 8.09 & 12.8 \\
\hline
\end{tabular}

Zeta potential of $\mathrm{R}$ solution is $23.2 \mathrm{mV}$.

The change of Zeta potential of PEI molecule was measure by Zeta sizer (Nano-ZS) from Malvern Instruments, as shown in the Table S2. The Zeta potential of PEI molecules with different molecular weights are all negative. After adding the resorcinol with a positive Zeta potential of $23.2 \mathrm{mV}$, the Zeta potential change from negative to positive charge, which further confirms that the resorcinol molecules could link with PEI molecules. 
Table S3. The relative contents of different functionalities in N 1s peaks from XPS spectra.

\begin{tabular}{ccccc}
\hline & \multicolumn{3}{c}{$\mathrm{N} 1_{\mathrm{S}}(\%)$} & $\mathrm{N} 4$ \\
\cline { 2 - 5 } Samples & $\mathrm{N} 1$ & $\mathrm{~N} 2$ & $\mathrm{~N} 3$ & - \\
\hline PMS-1 & 23.78 & 37.10 & 39.12 & - \\
PMS-2 & 21.07 & 38.57 & 34.36 & - \\
PMS-3 & 26.93 & 40.80 & 32.27 & - \\
PMS-4 & 31.41 & 52.58 & 16.01 & - \\
CMS-1 & 21.25 & 35.17 & 42.08 & - \\
CMS-3 & 22.08 & 38.31 & 37.19 & 21.72 \\
CMS-4 & 23.58 & 41.62 & 35.80 & 13.63 \\
ACMS3-2 & 23.10 & 33.82 & 21.36 & 28.29 \\
\hline ACMS3-3 & 22.83 & 35.25 & & \\
\hline
\end{tabular}

The relative contents of different functionalities in $\mathrm{N} 1 \mathrm{~s}$ are summarized in Table S3. For the carbonization samples, the relative contents of these different functionalities in $\mathrm{N} 1 \mathrm{~s}$ are similar. The graphitic N representing around $20-25 \%$ and almost no N-O is resolved. For the activated samples, it is clear that with the increase of the $\mathrm{KOH} / \mathrm{C}$ weight ratio, the relative content of the pyridinic $\mathrm{N}$ (N1) and pyridine-N-oxide (N4) decreases, while the relative contents of the pyrrolic/pyridone-N (N2) and the quaternary-N (N3) increase. 


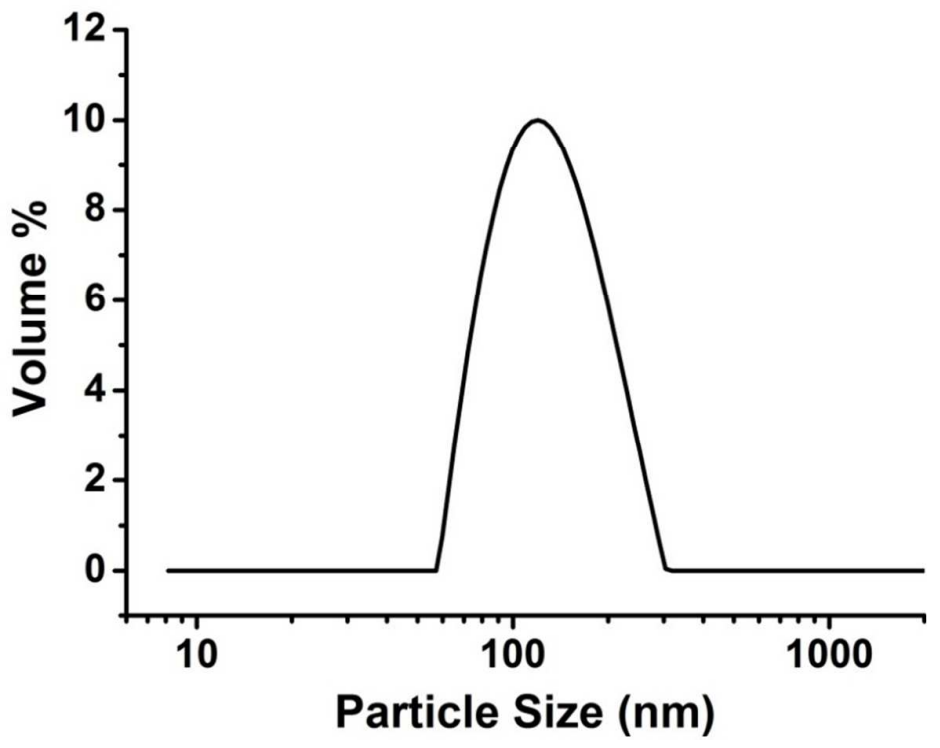

Figure S1. Particle size distributions of polymer microspheres synthesized with PEI-1800.

The particle size distribution of selected polymer microspheres synthesized with PEI-1800 is measured with the dynamic light scattering analyzer. It reveals that the particle size distribution is narrow with an average size of $122 \mathrm{~nm}$. 

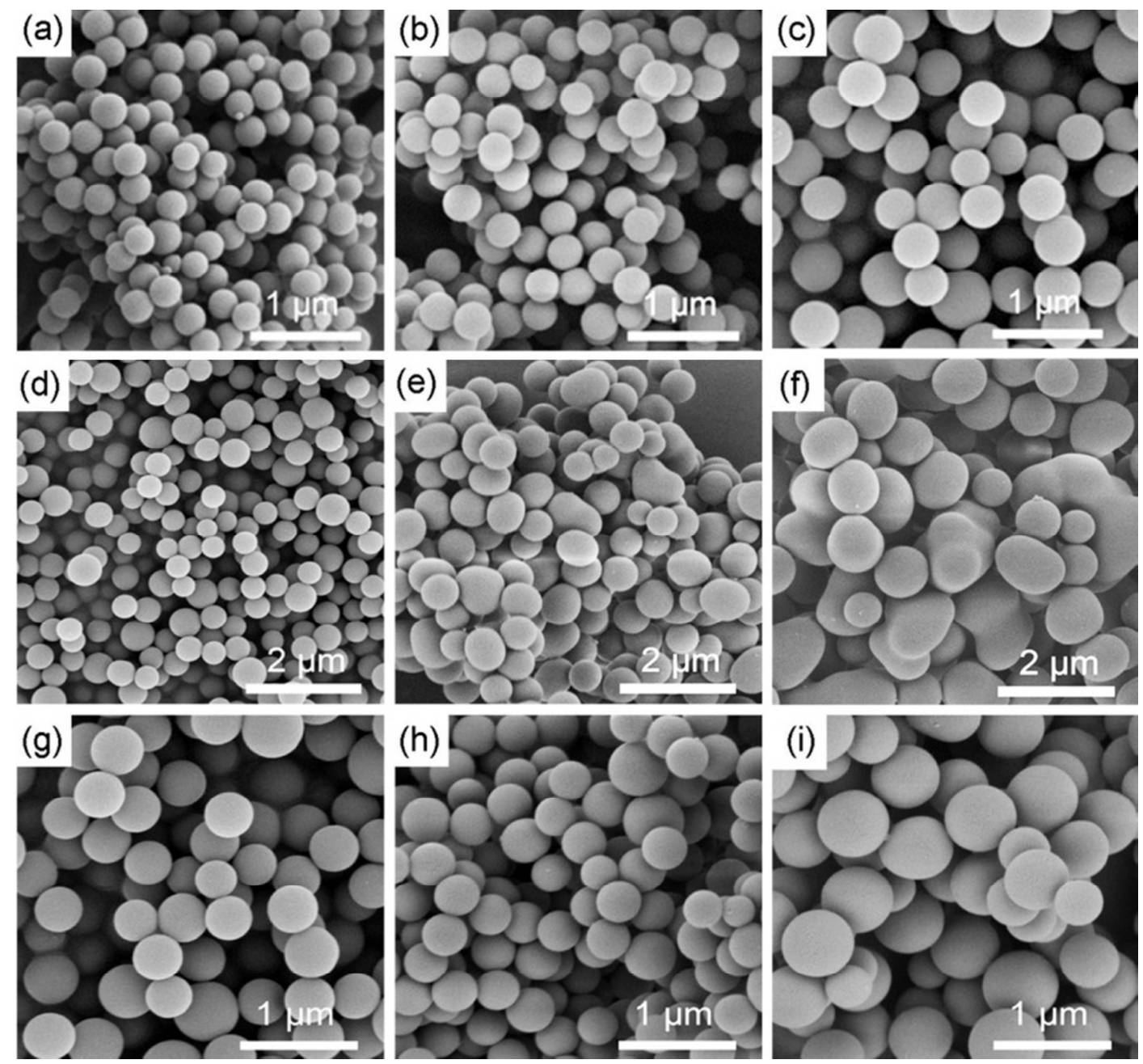

Figure S2. SEM images of PMS-PEI-600 obtained at different molar ratio of PEI/R: (a) 0.5; (b) 1; (c) 1.5; different concentration: (d)3 wt. \%; (e)5 wt. \%; (f)7 wt. \%; different temperature: (g)30 ${ }^{\circ} \mathrm{C}$; (h)50 ${ }^{\circ} \mathrm{C}$; (i)70 ${ }^{\circ} \mathrm{C}$.

In a typical synthesis process, the molar ratio of $\mathrm{PEI} / \mathrm{R}$ is 1.5 , the concentration is $3 \mathrm{wt} . \%$ and the temperature is $30{ }^{\circ} \mathrm{C}$. The series experiments mentioned above change only one experimental parameters each time. The SEM images of these PMSs demonstrate that relatively homogeneous microspheres can be obtained in a wide range of experimental operations. 

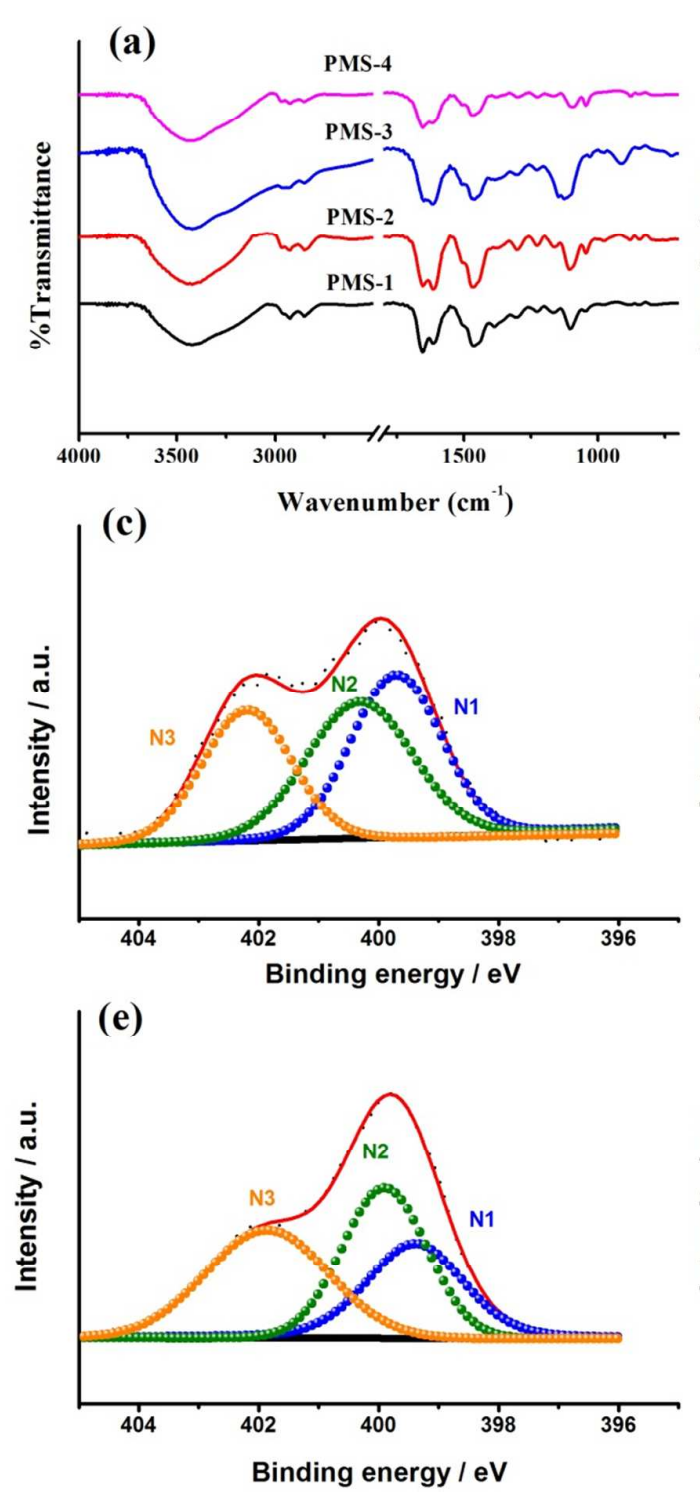

(b)
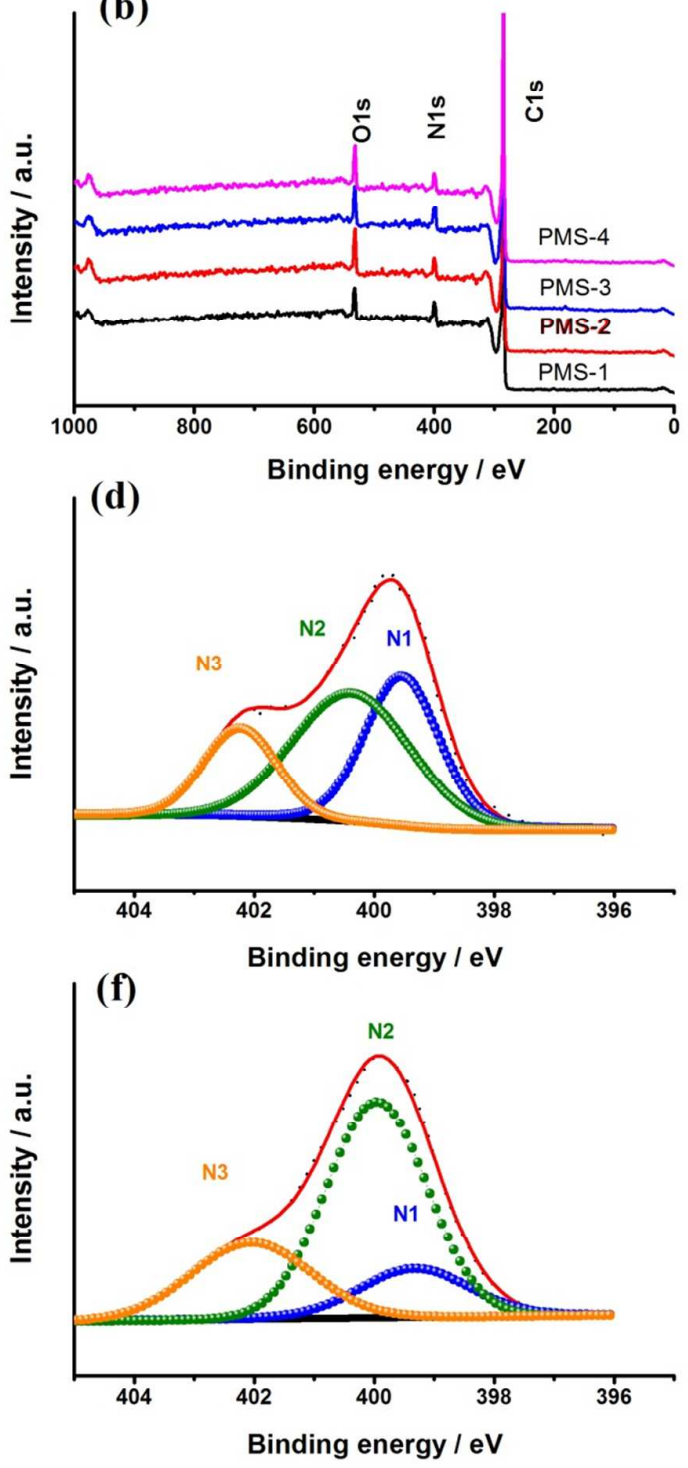

Figure S3. FT-IR spectra (a) and XPS survey (b) and high-resolution N 1s (c-f) spectra for PMSs synthesized of PEI with different molecular weight; (c) PMS-1; (d) PMS-2; (e) PMS-3 and (f) PMS-3.

In the FT-IR spectra showed in Figure S3 (a), the strong but broad band at $3430 \mathrm{~cm}^{-1}$ may be related to $\mathrm{N}-\mathrm{H}$ or $\mathrm{O}-\mathrm{H}$ stretching vibrations are observed for all samples. The bands at 1108 and $2926 \mathrm{~cm}^{-1}$ may be assigned to the $\mathrm{C}-\mathrm{O}$ and $\mathrm{C}-\mathrm{H}$ stretching of the polymer framework formed by polymerization of resorcinol, formaldehyde and PEI. Besides, the broad peak around $1104 \mathrm{~cm}^{-1}$ is assigned to the $\mathrm{C}-\mathrm{N}$ stretching 
vibration. To conclude, the FT-IR analysis suggests the existence of $\mathrm{N}-\mathrm{H}$ and $\mathrm{C}-\mathrm{N}$ species in the nitrogen-doped polymer samples.

The $\mathrm{N}$ 1s spectra are curve-fitted into three peaks with binding energies of $399.4 \pm 0.3,400.3 \pm 0.3$, and 402.1 $\pm 0.5 \mathrm{eV}$ that correspond to C-NH2 (N1), C-NH-C (N2), and C3-N (N3), respectively. It is worthwhile to note that the distribution of $\mathrm{N}$ species is different from each other while they have similar nitrogen content. This should be easily understood that the PEI with high molecular weight have relative small N1 and high ratio of $\mathrm{N} 2$. 


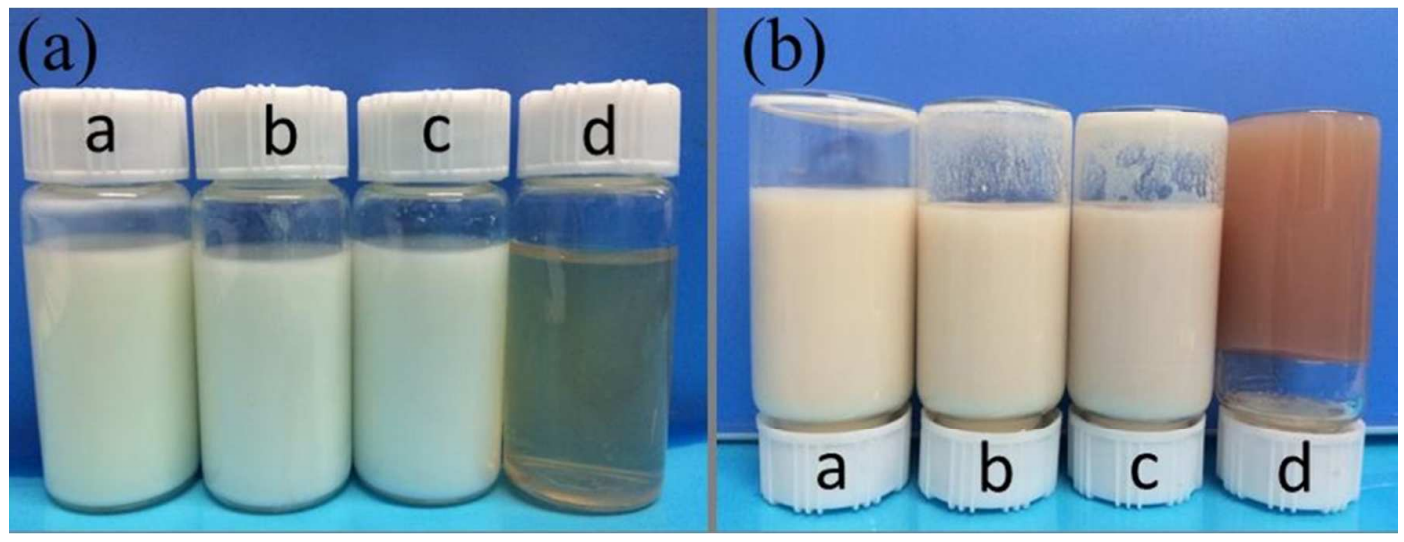

Figure S4. A digital photo image of the solution obtained with different molecular weight of PEI. a. PMS-1; b. PMS-2; c. PMS-3 and d. PMS-4; (a) after 30 min; (b) after $72 \mathrm{~h}$.

After adding formaldehyde into R-PEI solution, the polymerization reaction was very fast even under a room temperature. As shown in Figure S4, the reaction solution immediately becomes creamy white within $5 \mathrm{~s}$ after the addition of formaldehyde into PEI-resorcinol solution. In addition, the color of mixture solution becomes shallow gradually as the PEI molecular weight decrease, which indicates the decrease in reaction rates. After setting for 3 days, the solution did not change obviously except for PEI (10000), which experiences subsequent structure development towards gel formation. 


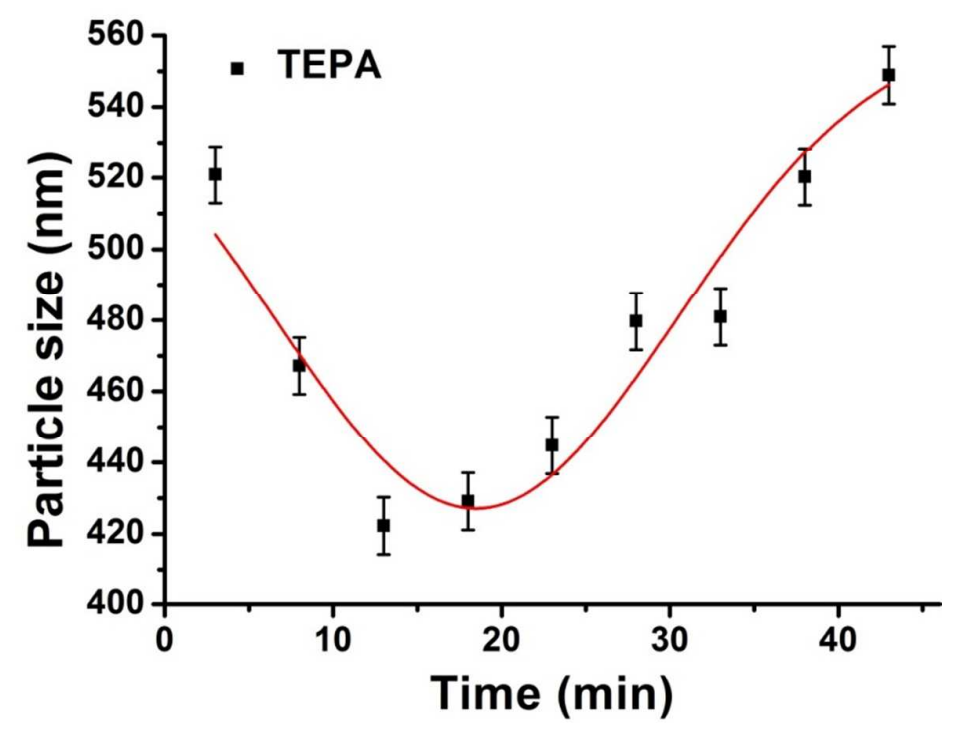

Figure S5. Transient changes of the hydrodynamic size of colloidal particles synthesized with TEPA

The shrinkage-growth process could be observed for all the organic amines used. Transient changes of the hydrodynamic size of colloidal particles synthesized with TEPA are shown in Figure S5, which gradually decrease to reach a minimum of $429 \mathrm{~nm}$ at $18 \mathrm{~min}$, and then follows an increase in size over time. 


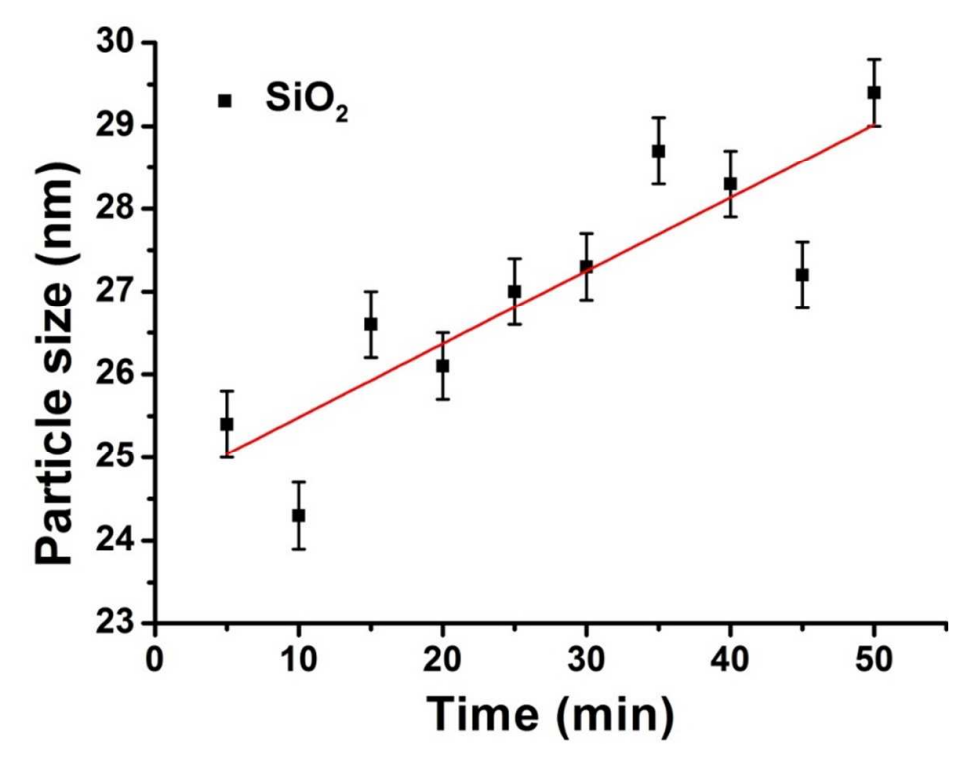

Figure S6. Transient changes of the hydrodynamic size of colloidal particles synthesized with TEOS using conventional Stöber synthesis method

The preparation procedure for colloidal $\mathrm{SiO}_{2}$ Particles: the reaction was facilitated by hydrolysisand condensation of TEOS in aqueous-alcoholic solution with ammonia $\left(\mathrm{NH}_{3} \mathrm{OH}\right)$ as the base catalyst. Typically desired amount of ammonia $(7 \mathrm{ml})$ was dissolved in an aqueous-alcoholic solution (mixing $65 \mathrm{~mL}$ of ethanol and $28 \mathrm{~mL}$ of distilled water). Subsequently, $4 \mathrm{ml}$ TEOS (ethylsilicate) was added slowly in the above solution with consecutive agitation. The reaction was conducted at room temperature.

A series of in-situ DLS experiments were performed to investigate the apparent mean hydrodynamic diameter of primary $\mathrm{SiO}_{2}$ clusters. The colloidal silica experience a gradual increase in size on the time responding to a typical Stöber process by continuous growth by diffusion of species form the solution towards the surface of nuclei. 

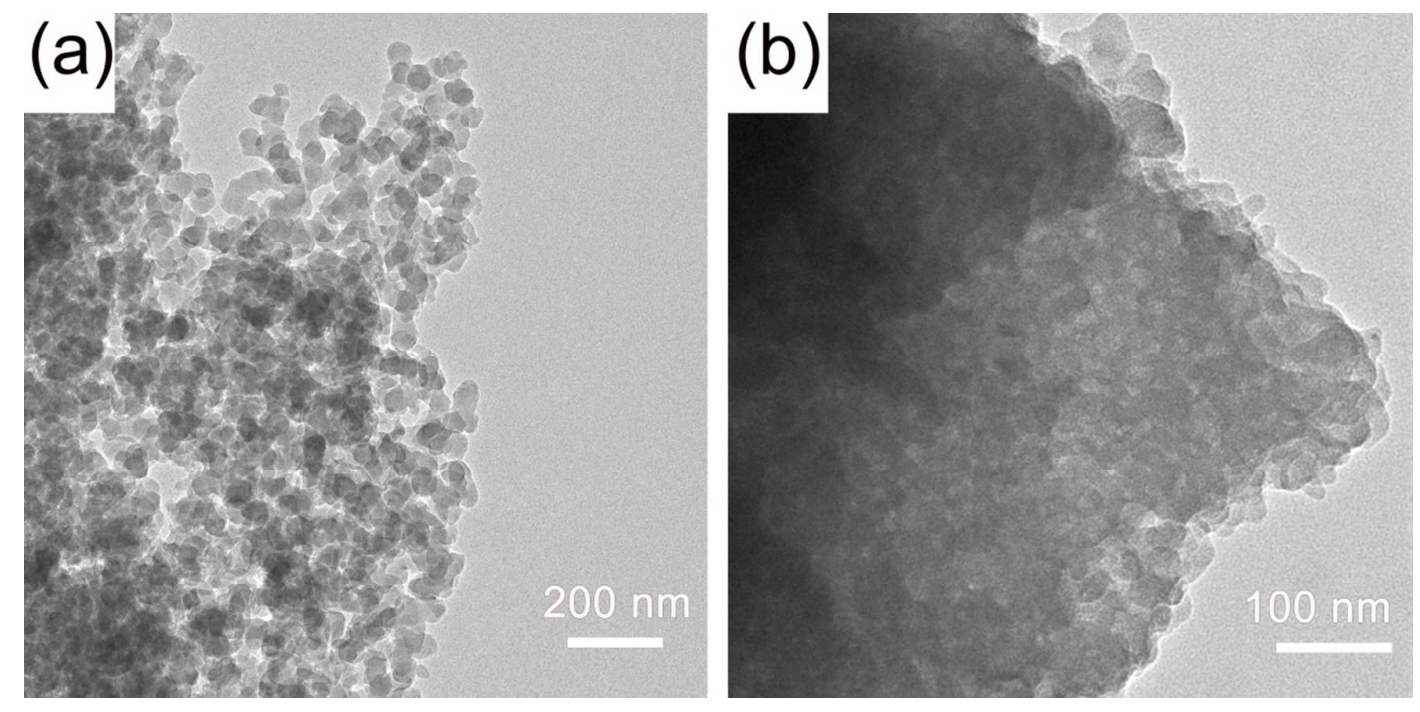

Figure S7. TEM images of PMS-4 (a) and CMS-4 (b).

In case of CMS-4, the polymeric nanoparticles are melted into condensed solid without on obvious porosity, and no core-shell structure could be found both in the polymeric and carbon samples 

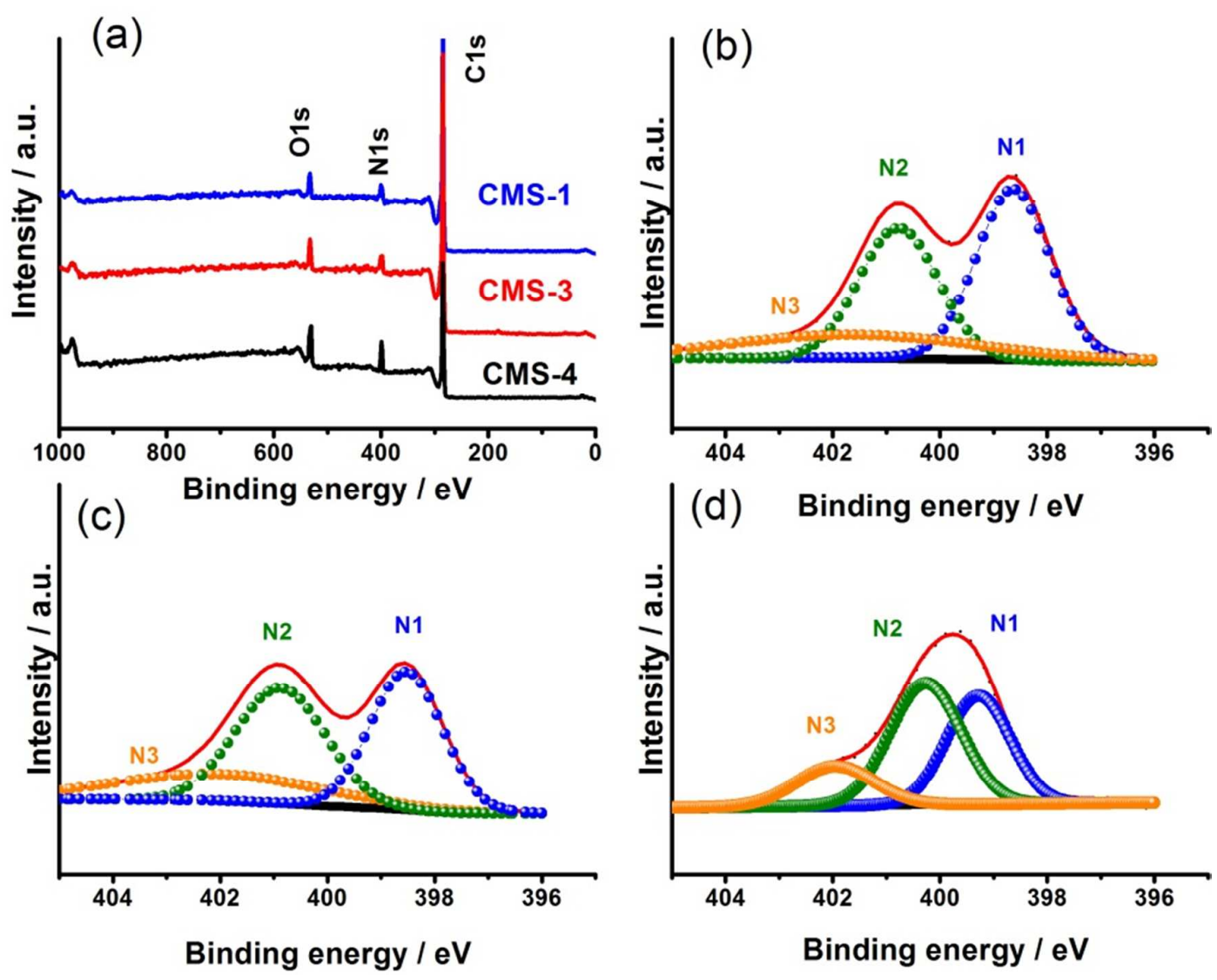

Figure S8. XPS survey (a, b) and high-resolution N 1s (c-d) spectra for CMSs synthesized from branched PEI; (b) CMS-1and, (c) CMS-3 and (d) CMS-4.

The relative contents of these different functionalities in $\mathrm{N} \mathrm{1s}$ are similar. The graphitic $\mathrm{N}$ represent around $21-25 \%$ and no $\mathrm{N}-\mathrm{O}$ is resolved. 

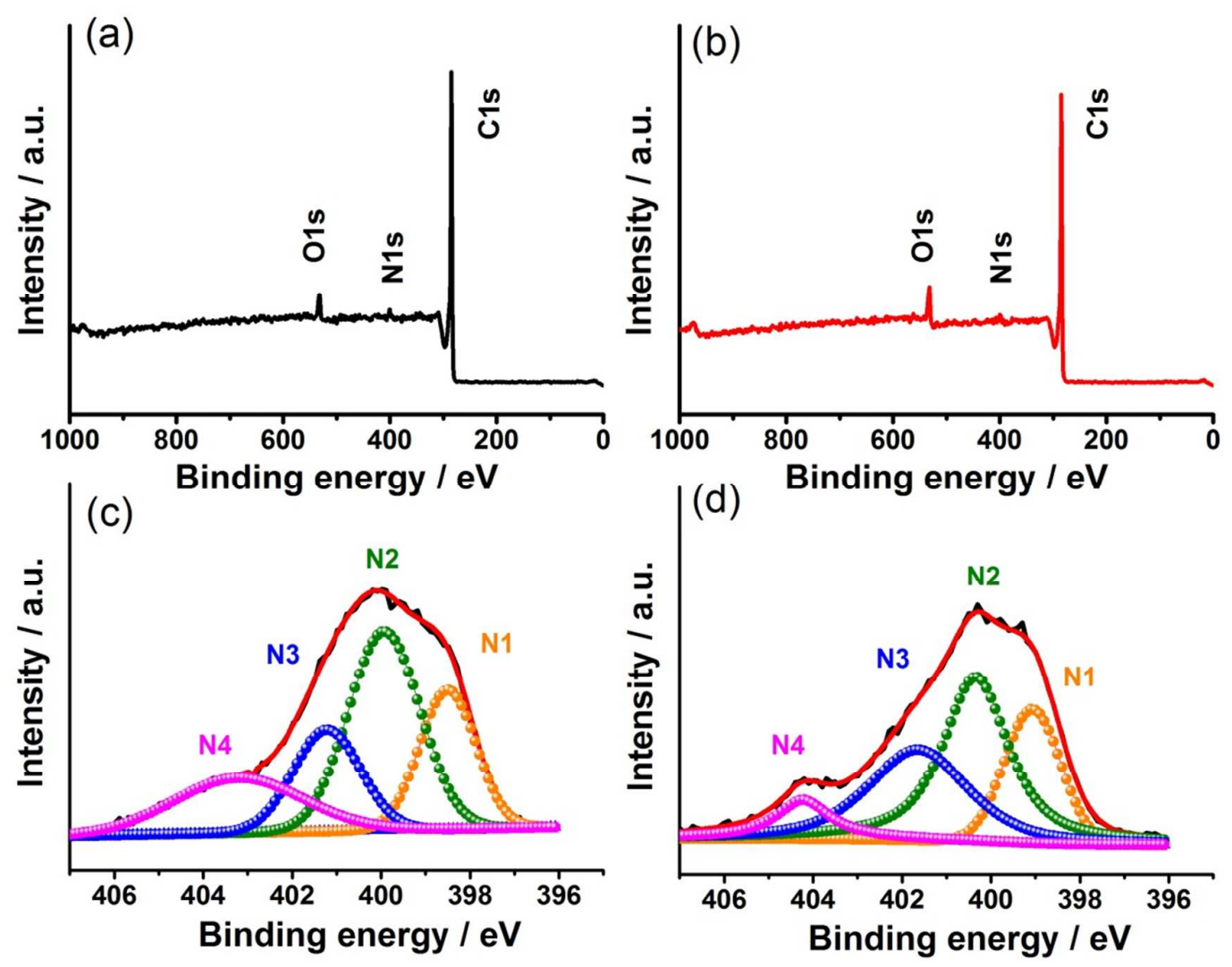

Figure S9. XPS survey (a, b) and high-resolution N 1s (c, d) spectra for ACMSs synthesised with branched PEI; (a, c) ACMS3-2 and (b, d) ACMS3-3.

The XPS results of ACMSs presented in Figure S9 (c-d) shows that the N 1s spectra are curve-fitted into three peaks with binding energies of $398.6 \pm 0.3,400.3 \pm 0.3,401.3 \pm 0.3$, and $404.0 \pm 0.3 \mathrm{eV}$ that correspond to pyridinic $\mathrm{N}(\mathrm{N} 1)$, pyrrolic $\mathrm{N}(\mathrm{N} 2)$, graphitic $\mathrm{N}(\mathrm{N} 3)$ and $\mathrm{N}-\mathrm{O}(\mathrm{N} 4)$, respectively. 

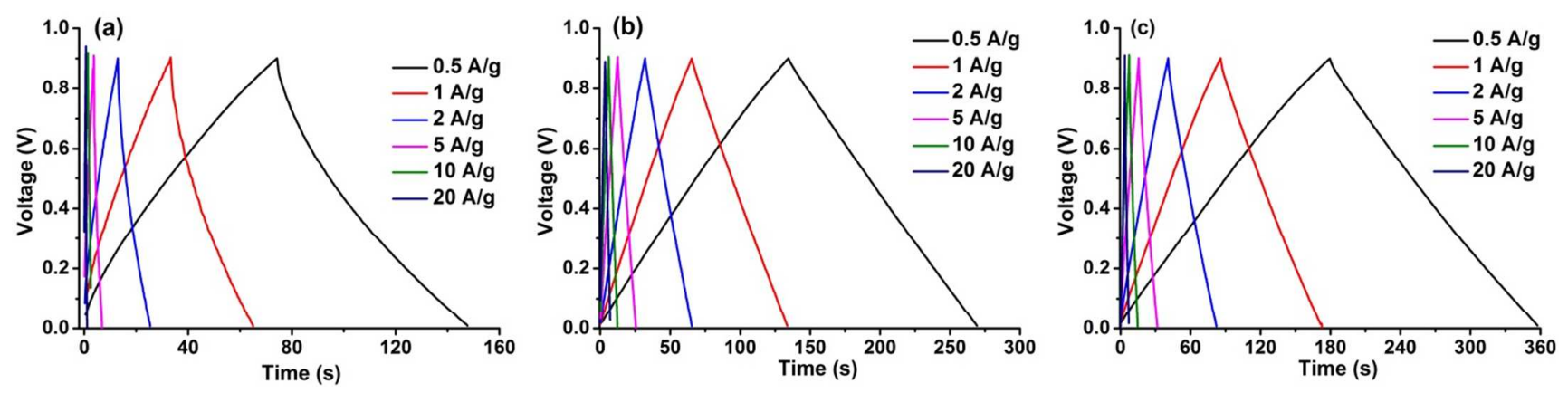

Figure S10. Galvanostatic charge/discharge voltage profiles at different current densities for CMS-3 (a), ACSM3-2 (b) and ACSM3-3 (c)

Figure S10 shows the galvanostatic charge/discharge curves of CMS-3 (a), ACSM3-2 (b) and ACSM3-3 (c) at different current densities $\left(0.1-20 \mathrm{~A} \cdot \mathrm{g}^{-1}\right)$. The shapes of the charge/discharge curves for all the samples are closely linear and symmetrical, indicating an excellent capacitive behavior and electrochemical reversibility. 

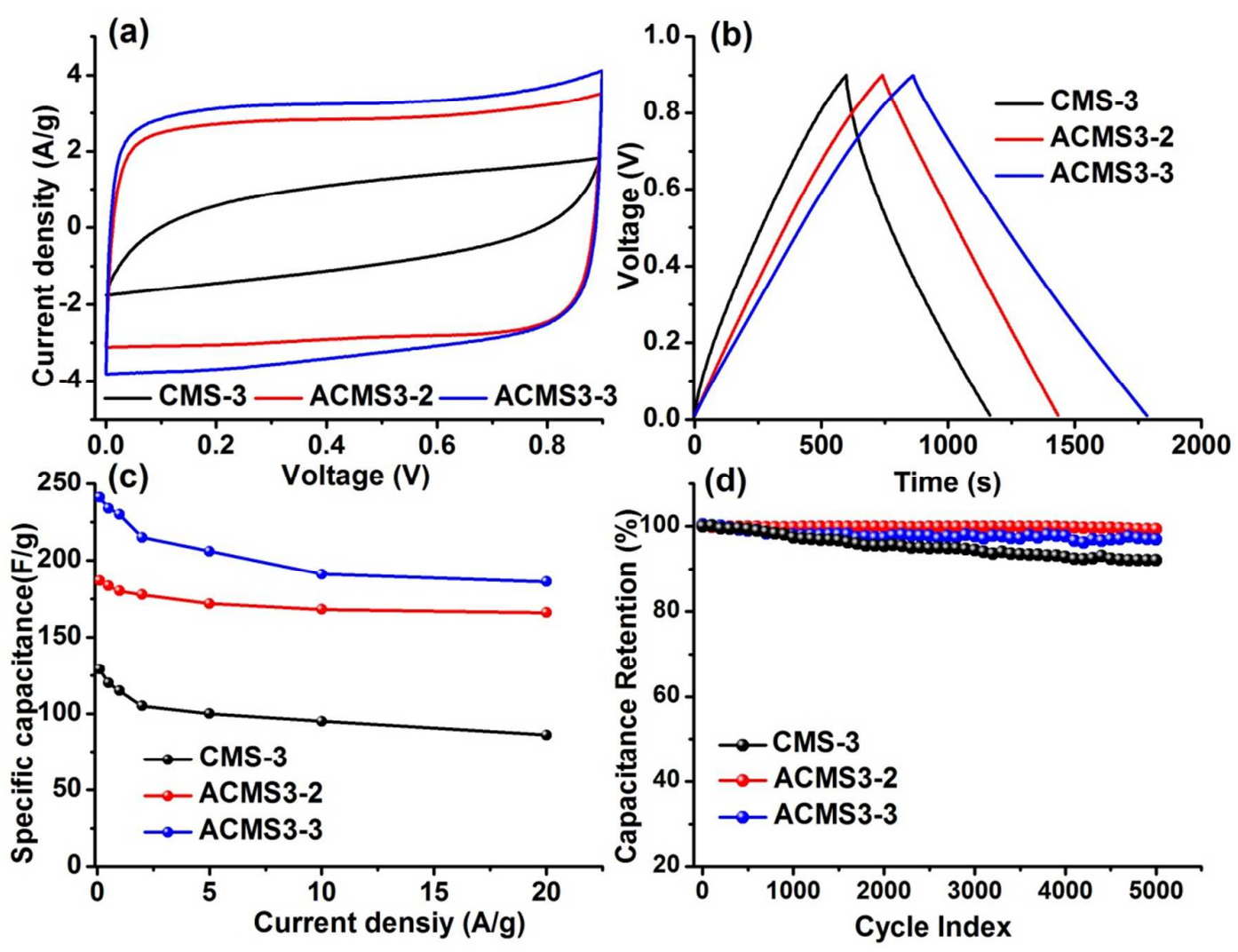

Figure S11. (a) cyclic voltammetry $(\mathrm{CV})$ testing at $100 \mathrm{mV} \cdot \mathrm{s}^{-1}$; (b) Galvanostatic charge/discharge voltage profiles at $0.1 \mathrm{~A} \cdot \mathrm{g}^{-1}$; (c) Plot of specific capacitances calculated from the discharge curves versus current density; (d) Cycling ability at $0.1 \mathrm{~A} \cdot \mathrm{g}^{-1}$ for CMS-3, ACMS3-2 and ACMS3-3.

The CMS-3 exhibits small rectangular curves corresponding to low capacitances due to the less developed pore structure. Moreover, the galvanostatic charge/discharge curve of CMS-3 is nearly symmetric with a gradual slope change, suggesting a typical capacitive performance. The specific capacitance of CMS-3 is only $129 \mathrm{~F} \cdot \mathrm{g}^{-1}$, much lower than the activated samples of 186 and $241 \mathrm{~F} \cdot \mathrm{g}^{-1}$ for ACMS3-2 and ACMS3-3, respectively. Moreover, CMS-3 could only retain a capacitance retention ratio of $70 \%$, lower than ACMS3-2 and ACMS3-3. In addition, the capacity decay is $9 \%$ for CMS-3, indicating a worse cycling stability than ACMS3-2 and ACMS3-3. 


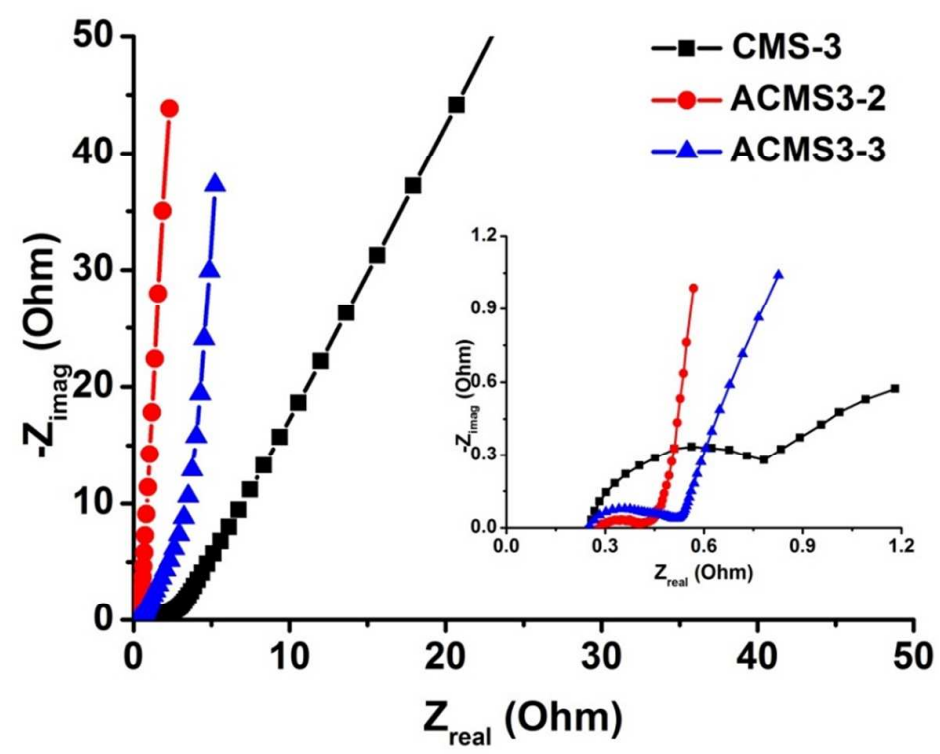

Figure S12. Nyquist plots and the enlargement of the high-frequency region (insert) for CMS-3, ACMS3-2 and ACMS3-3.

Each Nyquist plot is composed of a semicircle at high frequency and a nearly vertical line at low frequency. The intercept of the curve with the real impedance axis represents the equivalent series resistance (ESR), which is a key parameter in influencing the charge/discharge rate, as a smaller ESR value represents a lesser internal loss and a greater charge/discharge rate. The equivalent series resistance (ESR) value of ACMS3-2 and ACMS3-3 is about $0.14 \mathrm{Ohm}$ and $0.25 \mathrm{Ohm}$, respectively, much lower than CMS-3 of about $0.55 \mathrm{Ohm}$, indicating high charging and discharging rate of the activated samples. The vertical line indicates the ideal capacitive behavior of the cell. It is obvious that the line becomes much more vertical at low frequency of ACMSs than CMS-3, suggesting the superior capacitive behavior. 